\title{
Alter Ego : analyse de la réception d'une gamification artistique sur le Web
}

\section{Françoise Lejeune}

\section{(2) OpenEdition}

\section{Journals}

Édition électronique

URL : http://journals.openedition.org/sdj/319

DOI : $10.4000 /$ sdj.319

ISSN : 2269-2657

Éditeur

Laboratoire EXPERICE - Centre de Recherche Interuniversitaire Expérience Ressources Culturelles Education

\section{Référence électronique}

Françoise Lejeune, "Alter Ego : analyse de la réception d'une gamification artistique sur le Web», Sciences du jeu [En ligne], 2 | 2014, mis en ligne le 24 octobre 2014, consulté le 28 mars 2021. URL: http://journals.openedition.org/sdj/319 ; DOl : https://doi.org/10.4000/sdj.319

Ce document a été généré automatiquement le 28 mars 2021.

\section{(c) (i) (9)}

La revue Sciences du jeu est mise à disposition selon les termes de la Licence Creative Commons Attribution - Pas d'Utilisation Commerciale - Pas de Modification 4.0 International. 


\title{
Alter Ego : analyse de la réception d'une gamification artistique sur le Web
}

\author{
Françoise Lejeune
}

\section{Présentation du jeu artistique Alter Ego}

1 Alter Ego est un jeu artistique à réalité alternée ou ARG que j'ai impulsé en 2013. Cet ARG, qui s'est tenu à Metz le 5 avril 2013 et le 28 mars 2014, a été financé par l'université de Lorraine et réalisé en partenariat avec l'IUT de Saint-Dié, la ville de Metz, le Centre Pompidou Metz, les Galeries Lafayette et les associations étudiantes Cleb'art et TUM. En 2014, Alter Ego a couplé jeu en ligne et application de réalité augmentée sur des smartphones fournis par l'Université de Lorraine. Neuf équipes, de quatre, cinq ou six joueurs, se scindaient respectivement en deux, certains joueurs restaient derrière leur écran d'ordinateur pour résoudre les énigmes du jeu en ligne, créé par les étudiants de l'IUT de Saint-Dié dans les Vosges, les autres se rendaient dans les rues de Metz pour récolter des indices. La découverte des indices permettait d'élucider les énigmes, d'avancer dans les quêtes et gagner des diamants. Enfin, Alter Ego étant un rallye, le jeu favorisait la rapidité, l'équipe ayant réalisé le meilleur temps et gagné le plus de diamants gagnait le jeu. Alter Ego avait une double visée : le jeu entendait d'une part faire partager aux joueurs une aventure relationnelle quatre heures durant, amenant ainsi le joueur à prendre connaissance de ses capacités empathiques, il entendait d'autre part ménager des moments de pause, en vue de favoriser l'éveil de moments contemplatifs ou participatifs, selon le type d'œuvre présenté. Alter Ego attendait donc des joueurs une attitude ludique mais entendait également détourner l'attention cognitive des joueurs - en tant que ressource mentale mobilisée sur une ou plusieurs tâches - pour la transformer en attention esthétique, afin de marquer positivement l'expérience esthétique et l'associer à un moment de plaisir. En 2014, Alter Ego s'appuyait sur la fiction suivante : « Suite à une collision entre un météorite et notre soleil, celui-ci se dilate. L'air sur la surface de la Terre est devenu 
irrespirable et les Terriens se réfugient sous sa surface. Un siècle plus tard, l'un de ces Sous-Terriens est remonté à la surface, il a été kidnappé par des Terriens qui ont muté. Jusqu'où irez-vous pour le sauver?» français par jeux à réalité alternée ou jeux en réalité alternée. La préposition "en ", dans l'appellation jeux "en réalité alternée ", semble désigner le jeu selon le sens anglo-saxon de playing, soit le vécu subjectif du joueur, tandis que la préposition «à ", dans jeux "à réalité alternée ", semble renvoyer au jeu en tant que game, à ses règles objectives. La game-designer définit le jeu à réalité alternée comme " un drame interactif qui se joue à la fois en ligne et dans le monde réel » (Jenkins, 2013, p. 161). Je proposerai dans cet article d'étudier ce phénomène de gamification, en tant que contagion du jeu dans toutes les sphères de la société, dans sa dimension numérique et notamment artistique, à travers l'étude de cas du jeu Alter Ego.

En premier lieu, cette étude prendra pour cadre théorique la définition du jeu que donne Roger Caillois. Pour le sociologue français, il est possible de classifier les vécus subjectifs des joueurs en distinguant quatre "catégories fondamentales" (Caillois, 1958, p. 50) : l'Agon ou la compétition, l'Alea ou le jeu de hasard, la Mimicry ou le jeu de simulation et l'́linx ou la sensation de vertige. Nous essaierons de comprendre comment ces catégories fondamentales se sont articulées entre elles durant le jeu à l'étude. Plus près de nous, Jane McGonigal, dans son livre intitulé Reality is Broken, définit le jeu à la fois par ses éléments structuraux, le game, et par son vécu subjectif, le play (McGonigal, 2011). Le psychiatre Rémi Bailly fait remonter cette opposition entre game et play au pédopsychiatre anglais Donald $\mathrm{W}$. Winnicott qui plaçait le jeu au centre de ses analyses (Bailly, 2001, pp. 41-45). Concernant ses éléments structuraux, le jeu aurait selon McGonigal « un but, des règles, un système de retour d'information et une participation volontaire » (McGonigal, 2011, p. 21, traduction libre). McGonigal concède que cette définition omet « l'interactivité, le graphisme, la narration, la récompense, la compétition, les environnements virtuels ou l'idée de "gagner", de nombreuses caractéristiques que nous assimilons aux jeux contemporains. Il s'agit en effet d'aspects communs de beaucoup de jeux, mais qui ne définissent pas le jeu » (McGonigal, 2011, p. 21 , traduction libre). Pour l'auteure, les quatre caractéristiques du jeu précitées constituent le noyau d'un jeu, tout le reste n'est qu'effort pour renforcer ces quatre éléments fondamentaux. De même, Alter Ego avait un but clair, il s'agissait d'un rallye, l'équipe gagnante devait arriver avant les autres à la dernière étape du jeu. Alter Ego comprenait des règles : les joueurs en ligne devaient répondre à des énigmes tandis que leurs camarades récoltaient des indices dans les rues de Metz. Le retour d'information pour les joueurs en ligne, ou feedback, s'effectuait en temps réel, soit par le maître du jeu en 2013, soit grâce à un compteur numérique en 2014. Enfin, la participation des dix équipes de joueurs était bien volontaire. Techniquement, Internet, le jeu en ligne, l'échange de textos, la réalité augmentée et la téléphonie mobile étaient au cœur du dispositif. Mais Jane McGonigal définit également le jeu par sa réception, le play. Se référant à Chris Bateman, McGonigal écrit que le jeu peut être un simple divertissement ou éveiller des émotions plus fortes comme le fiero que "nous exprimons presque tous de la même manière: nous levons les bras au-dessus de notre tête et hurlons " (McGonigal, 2011, p.47, traduction libre) et le naches ou fierté pour autrui ${ }^{1}$ (McGonigal, 2011, p. 101). Dans le meilleur des cas, le jeu peut mener à l'expérience optimale décrite par Mihály Csíkszentmihályi : " "Les jeux sont une source évidente de flot”, a-t-il écrit, 
“et le jeu est l'expérience de flot par excellence".» (cité par McGonigal, 2011, p. 50, traduction libre). ${ }^{2}$

\section{Méthode de recherche}

Des questions de recherche se posent. Le jeu est-il parvenu à ménager des moments contemplatifs et empathiques ? Ou, au contraire, l'expérience des joueurs n'a-t-elle été que pur plaisir ludique ? La compétition a-t-elle nui à la collaboration entre équipes adverses ou bien des moments de partage ont-ils pu émerger malgré cette compétition?

5 J'analyserai la gamification artistique sur le réseau à travers un corpus méthodologique comprenant :

- l'observation en présentiel de l'activité des joueurs dans la salle informatique ;

- des enregistrements vidéo de l'activité des joueurs dans la salle informatique et dans la rue ;

- un enregistrement des textos que se sont adressés les joueurs via l'application Alter $\mathrm{Ego}^{3}$;

- une enquête sur les émotions inspirée de la Question 33 du Questionnaire Genevois d'Appraisal ou GAQ de Klaus Scherer associée à une question sur le moment fort du jeu ${ }^{4}$;

- des entretiens réalisés auprès de 37 joueurs, dont 15 joueurs en ligne.

6 Je présenterai dans un premier temps le champ des ARG artistiques avant de présenter plus précisément les modalités d'immersion dans le jeu. Je partagerai certains résultats de ma recherche en m'attachant particulièrement à décrire le vécu psycho-social des joueurs en ligne, lorsque cela est possible. Cela me conduira à discuter des conditions de l'émergence de moments contemplatifs durant le déroulement du jeu.

\section{Blast Theory : les fondateurs}

7 La gamification renvoie au terme anglais game et pourrait ainsi, plus précisément, se référer à l'aspect structural du jeu. Dès leurs premiers jours, les ARG artistiques ont tenté de transformer cette gamification en expérience du vivre ensemble. Nous devons cette gamification de l'œuvre d'art au collectif d'artistes britannique Blast Theory qui a fait des débuts remarqués dans les années 1990 avec un théâtre participatif. En 1991, le collectif créait Gunmen kill three, une pièce de théâtre inspirée d'un article du quotidien The Guardian, intitulé « Des hommes armés tuent trois personnes dans une boutique de téléphones mobiles ». L'histoire était la suivante : deux agresseurs protestants étaient entrés dans un magasin de téléphonie mobile pour tuer, en criant «IRA», deux femmes derrière le comptoir ainsi qu'un homme qui tentait d'intervenir. La pièce de théâtre proposait une reconstitution de l'assassinat dans le magasin, la mise en scène comprenait des projections vidéo tandis qu'un pistolet de paintball était remis à un spectateur afin qu'il tire sur les interprètes. Ainsi, Blast Theory ne proposait pas au spectateur de se représenter un assassinat commis par une tierce personne mais d'en faire l'expérience en première personne. En 1998, Blast Theory allait un peu plus loin dans la participation. Avec Kidnap, le jeu de rôle fit définitivement son entrée dans le vocabulaire artistique du collectif qui proposa un tirage au sort à l'issue duquel les gagnants vivraient une expérience de l'enlèvement. Parmi les dix finalistes, en Angleterre et au Pays de Galles, deux gagnants, choisis au hasard, furent placés sous surveillance et enlevés en plein jour. Ils furent emmenés dans un lieu secret, où ils 
furent détenus pendant quarante-huit heures, sous l'œil vigilant d'une psychologue. L'ensemble fut diffusé en direct sur Internet grâce à une webcam qui filma en continu les deux prisonniers tandis que les internautes communiquaient en direct avec les ravisseurs. Stephen Armstrong, après son enlèvement, témoignait : " Mon expérience de la performance a été assombrie par la terreur, la frustration, l'ennui et la colère qui a dominé mes 24 heures de captivité. [...] Aucun autre spectacle que j'ai vu n'a provoqué une telle intensité d'émotions $»^{5}$. Depuis, Blast Theory n'a cessé de questionner les outils numériques. Comme le rappelait Bernard Guelton dans la revue Interfaces numériques en 2012, " la démarche artistique des artistes de Blast Theory est au croisement de trois courants qui sont le théâtre participatif, la performance et l'art en réseau appareillé » (Guelton, 2012, p. 58). Le virage ludique du collectif est pris en 2001 avec Can You See Me Now? Le jeu, réactivé en 2005 à Tokyo, consiste en une chasse à l'homme par des internautes qui naviguent dans un univers virtuel en trois dimensions reproduisant les rues de la ville. Pour cela, l'internaute communique avec un complice dans la rue. Des cibles vivantes sur le terrain sont munies de matériel de géolocalisation permettant à l'internaute de les repérer sur un écran. Bernard Guelton envisage dès lors la réception du jeu à réalité alternée du point de vue de l'immersion réelle. Pour ma part, je proposerai d'aborder l'ARG artistique Alter Ego par son immersion virtuelle et fictionnelle.

\section{Le développement des ARG artistiques en France : le cas des Mystères de la Basilique}

8 En France, les jeux à réalité alternée se multiplient. En 2009, à l'occasion de Lille 3000, la société Fabernovel et l'opérateur éditeur sur mobile SFR ont créé le jeu à réalité alternée Can You Stop It? dont le thème était l'arrivée mystérieuse de statues mi-anges mi-démons rue Faidherbes, à Lille. Les joueurs devaient sauver Lille de la destruction. Différentes technologies dont le flashcode, la borne Bluetooth, le serveur vocal et les SMS permettaient de résoudre les mystères et découvrir les indices cachés dans la ville. En 2012, à la demande de la Basilique de Saint-Denis et de la ville de Saint-Denis, Karleen Groupierre et Edwige Lelièvre ont créé un jeu de médiation patrimoniale intitulé Les Mystères de la Basilique, qui comprenait un jeu en ligne, Ghost invaders, coordonné avec des actions dans le monde tangible. Le jeu a duré un mois, 500 joueurs se sont inscrits au jeu en ligne et certains y ont joué plus de cinquante heures par mois (Lelièvre, 2012, p. 323). Les auteures du jeu ont présenté les résultats de leur recherche lors du colloque Ludovia, en 2012 (Groupierre et Lelièvre, 2013). Les Mystères de la Basilique reposaient sur une fiction : le fantôme du roi Dagobert réclamait son sceptre disparu sur fond de prophétie et de menace. Afin de pouvoir répondre à cette demande, les joueurs devaient continuellement se référer à la réalité historique, culturelle et patrimoniale de la ville de Saint-Denis. Pour ces auteures, les ARG trouveraient leur origine dans les jeux de rôle et les jeux de rôle en ligne ou Massively Multiplayer Online Role Playing Game (MMORPG) soit, en français, Jeu de Rôle en Ligne Massivement Multijoueur. Les auteures insistent sur le concept de transfiction, inventé par Christy Dena en 2009 , qui qualifie une fiction qui se déploie sur plusieurs supports, dépendants les uns des autres tels des journaux, des cartes de visite, de fausses publicités, un site Internet, etc. La transfiction a donc besoin de toutes les pièces, de tous les supports, 
pour être complète et resserre en cela, selon les auteures, la définition donnée par Henry Jenkins de l'histoire transmédia.

En effet, «En 2006, écrivent-elles, Henry Jenkins, dans son livre Convergence Culture pointe du doigt le terme de "transmedia storytelling". Dans cet ouvrage, il explique qu'une "histoire transmédia se déploie au travers de multiples supports, avec chaque nouvel élément apportant une contribution caractéristique et précieuse à l'ensemble". » (Groupierre et Lelièvre, 2013, p. 3). Cette définition permet de regrouper plusieurs types de transmédia. La transfiction, pour sa part, tisse un lien fictionnel entre tous les supports dont les contenus, une fois rassemblés, ne composent qu'une seule et même fiction. Karleen Groupierre et Edwige Lelièvre étudient ensuite les éléments des jeux de rôle en ligne réutilisables dans les ARG et, s'appuyant sur les analyses de McGonigal dans Reality is Broken, proposent trois clés d'immersion dans les jeux en ligne : la progression de l'avatar, le défi relevé lors des quêtes et l'aspect multi-utilisateurs de ces jeux. « La collaboration et le partage y sont donc tout à fait importants, concluent-elles, et sont une source importante de motivation.» (Groupierre et Lelièvre, 2013, p. 5) Dans sa thèse de doctorat, Lelièvre insiste en particulier sur le fait que les MMORPG comprennent de nombreux temps morts qui pourraient être mis à profit afin de favoriser l'éveil de moments contemplatifs (Lelièvre, 2012, p. 71). C'est sur ce dernier point qu'elle insista, lors d'un entretien qu'elle m'a accordé en 2013, me mettant en garde contre le danger de l'esprit de compétition qui pouvait nuire à la contemplation ${ }^{6}$. Elle expliquait ainsi que «le seul souci est plutôt l'aspect compétitif, qui là est autre chose, qui a des fois tendance à faire courir les joueurs, et du coup quand ils courent ils n'observent pas beaucoup, ils ont tendance à aller à l'intérêt ludique le plus important plutôt que d'apprécier réellement l'espace qui leur est offert. »

\section{L'immersion fictionnelle et virtuelle des joueurs d'Alter Ego}

10 Alter Ego s'est tenu de 13 heures à 17 heures, rassemblant 15 des 37 joueurs dans une salle informatique de l'Université de Lorraine. Je les ai observés et filmés, les textos échangés par les joueurs ont été enregistrés grâce à l'application Alter Ego pour smartphones. Il semble difficile, dans le cas particulier d'Alter Ego, de séparer la structure $\mathrm{du}$ jeu de sa réception. Nous attendions des joueurs, dont la participation était volontaire, une attitude ludique susceptible de les maintenir dans le jeu quatre heures durant. Afin d'y arriver, nous avons mis l'accent sur l'immersion fictionnelle, mais aussi sur l'immersion ludique en proposant des énigmes difficiles à résoudre si ce n'est grâce à la recherche documentaire sur le web.

11 Concernant l'immersion fictionnelle, sur chaque nouvelle page, correspondant à une nouvelle quête, une vidéo venait résumer la fiction. Sara, une joueuse en ligne de l'équipe des Corgi Cookies, explique : « Nous, dans la salle info, la fiction ça allait grâce aux vidéos. Les vidéos nous ont aidées. » L'équipe des Tryharders, qui découvre le texte de la fiction, écrit dans son texto : «Notre complice recherche un endroit protéger possible un garage ou sous sol » (sic). Cet abri était matérialisé dans la rue par une cabane de 5 mètres de long construite par des étudiants en Arts plastiques à l'intérieur de laquelle un indice les dirigeait vers l'étape suivante. Les internautes guidaient ainsi les joueurs sur le terrain en s'appuyant sur le récit de la fiction. Les échanges de textos montrent également que les joueurs ont contemplé les vidéos activement jusqu'à la 
quête 3, car ils espéraient y trouver des indices. Ils écrivent : «Dans la vidéo on voit un gars qui sort d'un garage avec une tête de cheval. Essaie d'entrer cheval. » $\mathrm{Si}$, pour les joueurs experts en art, ces vidéos ont été utiles à l'immersion fictionnelle, pour la plupart des joueurs, elles se sont avérées décevantes. Les réponses aux questionnaires montrent que seulement $42 \%$ des joueurs se sont appuyés sur les vidéos pour entrer dans la fiction. Pour la majorité des joueurs, l'attente aura été déçue : un étudiant en informatique écrit que les vidéos ne l'ont pas particulièrement marqué, un autre en sciences du langage parle de "perplexité ». Les statistiques concernant le nombre de clics sur chaque vidéo sont sans appel; le nombre de clics pour la première quête est égal à 24 vues, dès la quête 2 il chute à 10 vues pour ne plus compter que 4 vues dès la quête 4. Il n'y a majoritairement pas eu d'immersion fictionnelle dans les vidéos, en tant que rupture avec ce qui constitue le monde actuel des joueurs. Le faible succès des vidéos présentées pourrait s'expliquer en partie par le fait qu'elles étaient de simples illustrations de la fiction alors que le contexte de leur contemplation était de type compétitif. En effet, la salle est restée plutôt silencieuse durant tout le jeu, les joueurs comparaient constamment leurs scores et se surveillaient mutuellement. Au bout d'une heure de jeu, le stress était visible, les joueurs se rongeant les ongles, passant leurs mains sur leur visage et soufflant. Si nous reprenons les catégories de Caillois, il semblerait que l'accentuation du pôle compétitif dans la salle, l'Agon, se soit fait au détriment des autres dimensions du jeu, notamment de la Mimicry, à savoir l'entrée dans la fiction. Ainsi, une première analyse du jeu laisserait penser qu'à l'avenir, pour que les vidéos deviennent efficaces, celles-ci devraient s'adapter au genre dans lequel elles s'intègrent et participer d'une manière ou d'une autre au dynamisme du jeu.

Si l'immersion fictionnelle a peu fonctionné pour les joueurs en ligne, il en va autrement de l'immersion virtuelle. Serge Tisseron, pédopsychiatre, explique dans son essai Du livre et des écrans que l'immersion virtuelle repose sur l'interactivité et le fait que celle-ci nous permet de plier les objets numériques selon nos quatre volontés (Tisseron, 2013). En nous permettant d'interagir avec les objets et les images, l'interactivité nous permet également de construire nos propres repères pour prendre possession de l'espace dans lequel ces objets et images évoluent. Nous pouvons donc devenir spectateurs de nos propres actions en temps réel, simuler une action et mesurer les conséquences de nos actes. Une immersion virtuelle a également été proposée aux joueurs d'Alter Ego grâce à un jeu interactif, Hertzian Labyrinth, créé sur Processing par Jean-Michel Rolland. Il constituait la quête finale du jeu et débouchait sur une page indiquant aux joueurs qu'ils devaient se rendre au Centre Pompidou Metz. Il s'agit d'un jeu développant, selon son auteur, les capacités d'écoute du joueur. Ce jeu proposait aux joueurs de s'échapper d'une succession de salles dans lesquelles ils étaient enfermés. Ils commençaient le jeu dans la salle dont la fréquence sonore était de 150 Hertz, devaient trouver la clé qui se trouvait dans la salle dont la fréquence était de 486 Hertz et retourner dans la salle à 150 Hertz pour sortir victorieux. L'effet sonore était visualisé par la constitution d'un cercle brun devenant blanc lorsque les fréquences étaient synchronisées. 
Jean-Michel Rolland, Hertzian Labyrinth, capture écran
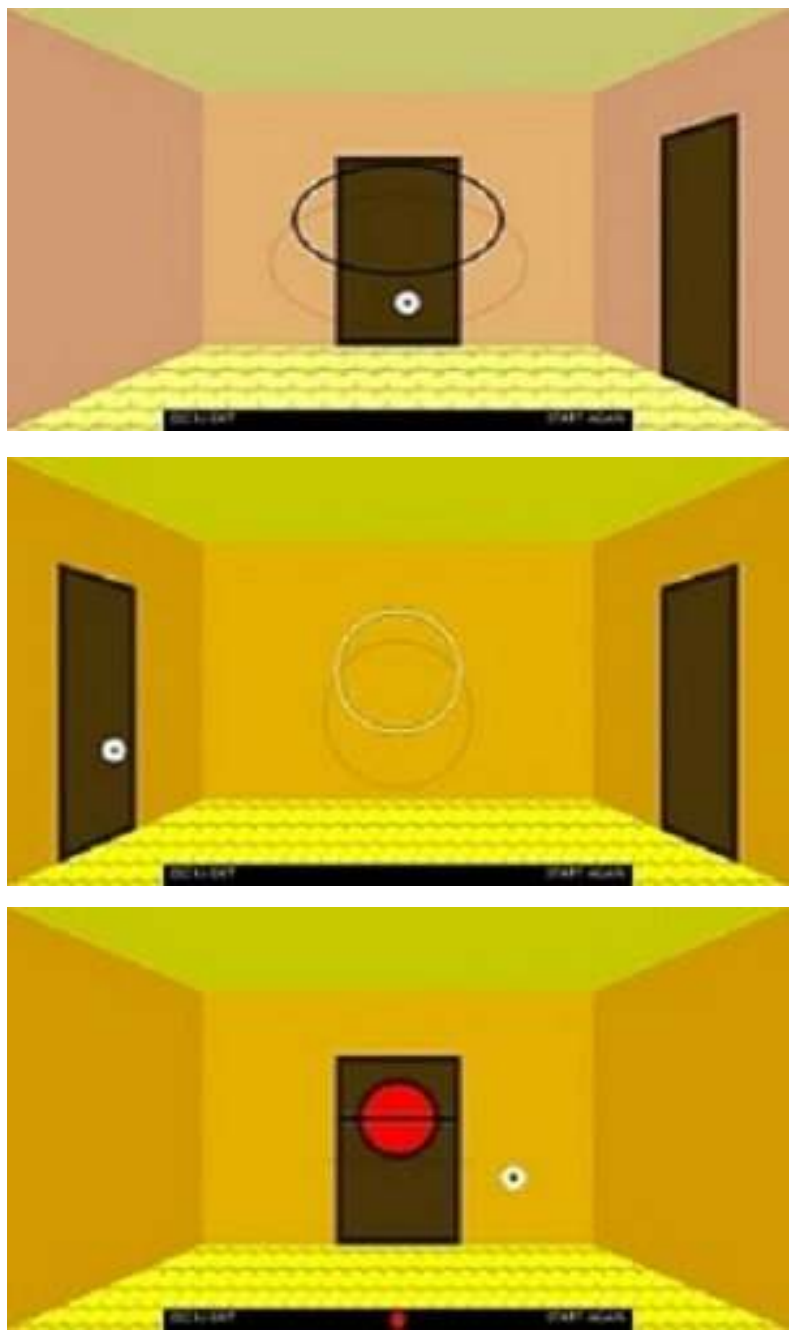

Date de sortie : 28 mars 2014.

13 Hertzian Labyrinth n'a pas laissé les joueurs indifférents, contrairement aux vidéos expérimentales, et a fait l'objet de nombreux commentaires. Les joueurs des Tryharders commentaient par texto la prise en main du jeu qu'ils trouvaient bizarre :

0 . « Aucune idée ptete qu'on aurra la réponse quand on finira le jeu bisarre »

1. « cest quoi votre jeu »

0 . « Ben je sais pas c'est un truc qu'on a eu après avoir fini le questionnaire c'est une sorte de labirinthe et faut utiliser le son apparement » (sic)

14 Les réponses aux questionnaires remis aux joueurs lors de la remise des prix indiquent que Herzian Labyrinth a fait partie des moments forts du jeu pour $15,78 \%$ des joueurs en ligne. Il fut également pour Guillaume, l'internaute de l'équipe des Moron Rabbits, l'occasion d'exprimer un sentiment de fiero, quant il sortit le premier du labyrinthe. Le joueur exprima sa fierté par un cri de victoire et en levant les bras en l'air. Contrairement aux vidéos, Hertzian labyrinth a bien été le lieu d'une immersion. Pour la première fois, après trois heures de jeu, Guillaume des Moron Rabbits dût couper le contact avec ses partenaires dans la rue et raccrocher son téléphone pour mieux se concentrer. Afin de se situer dans le labyrinthe, il était nécessaire d'élaborer une carte mentale, de se situer dans l'espace virtuel afin de ne pas reprendre deux fois le même chemin. Guillaume me demande très justement «si j'imagine que le labyrinthe est 
rectangulaire, la sortie du labyrinthe n'est pas au milieu?» Il sera le premier à en trouver l'issue. Les joueurs des Tryharders, qui sont venus avec leur ordinateur portable, jouent à deux à ce jeu. Ils échangent leur stratégie, l'un déclare « Il faut retenir le chemin par lequel tu passes », l'autre lui répond «C'est pas grave, tu peux toujours sortir et recommencer ». Le premier insiste : «On aurait dû l'écrire sur un bout de papier. " L'extrême attention requise par le déplacement dans le labyrinthe virtuel, l'interactivité permettant d'ouvrir et fermer les portes, la motivation des joueurs à gagner cette dernière étape expliquent l'immersion dans le jeu.

\section{Jean-Michel Rolland, Hertzian Labyrinth, plan du labyrinthe}

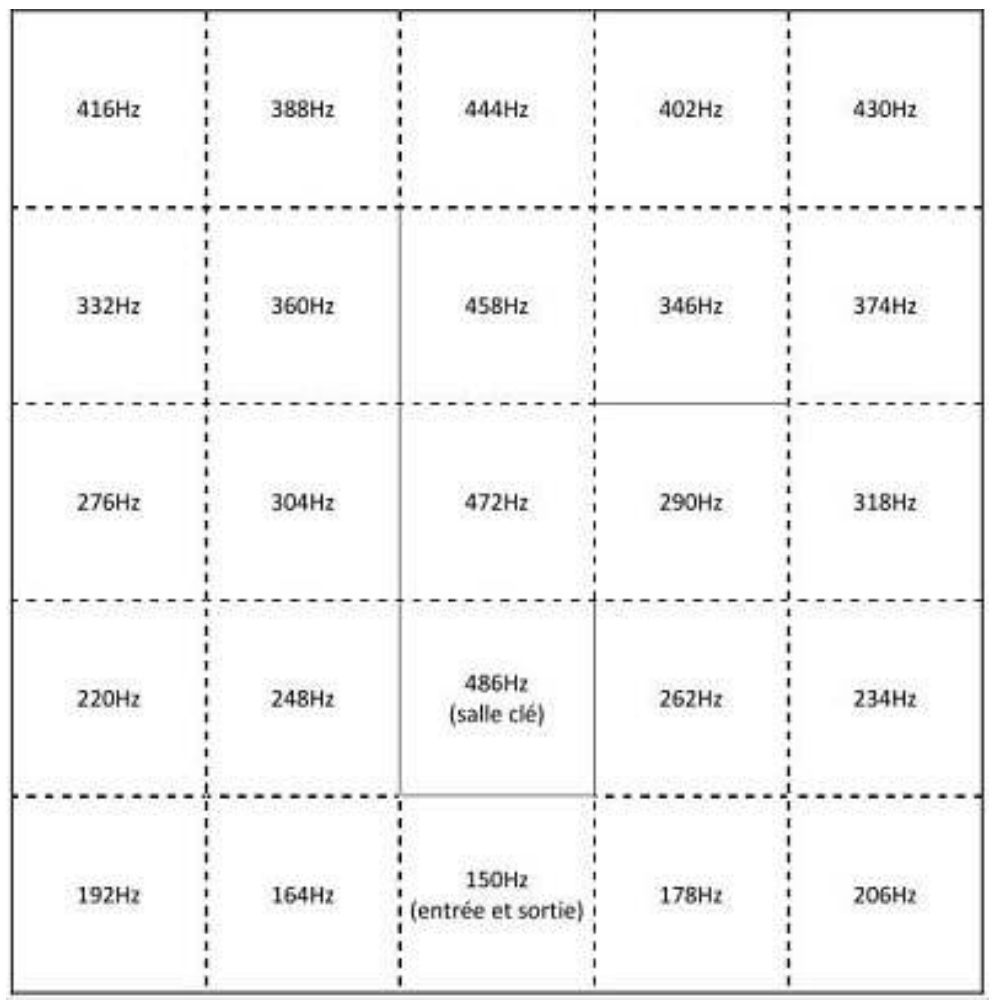

\section{Place de la contemplation dans la réception du jeu}

Alter Ego ne visait pas uniquement une réception ludique du dispositif mais entendait également détourner le jeu de sa visée non utilitaire pour éveiller des moments contemplatifs chez les participants. L'idée était de détourner l'attention cognitive des joueurs de leur cible pour la transformer en attention esthétique. À cette fin, nous avions ménagé des moments de pause. Edwige Lelièvre explique ainsi dans sa thèse que "Créer des temps de repos pour encourager les joueurs à être ensemble et à échanger est le conseil que donne Raph Koster, l'un des concepteurs de jeux de rôle en ligne les plus connus et l'un des précurseurs du genre dans ses "Lois du design des mondes en ligne" " (Lelièvre, 2012, 71). Les moments de repos que nous avions prévus devaient se dérouler pendant que les joueurs en ligne attendaient que leurs camarades sur le terrain se rendent à pied vers l'étape suivante. Cependant, comme en témoignent les observations et les échanges de textos, les joueurs n'ont pas procédé ainsi. Les cohortes ont préféré, la plupart du temps, se désynchroniser pour résoudre chacune de leur côté 
les énigmes. Les observations montrent que, dans la salle informatique, les quatre heures de jeu se sont déroulées de façon intense, sans temps mort, aucun joueur n'ayant souhaité quitter la salle pour faire une pause. Cette intensité du jeu peut s'expliquer par le fait qu'Alter Ego était un rallye, la première équipe arrivée à l'étape finale et ayant réussi toutes les quêtes sans malus gagnant le jeu. Ainsi, d'un point de vue stratégique, la rapidité comptait. Dans ce contexte, les joueurs ont voulu gagner du temps, par souci d'efficacité. Puisque les vidéos n'étaient pas vitales pour le jeu, elles n'ont été que très peu visionnées.

Or, si nous voulons détourner l'attention cognitive des joueurs pour la transformer en attention esthétique, nous devons tenir compte de ce sur quoi l'attention des joueurs se pose. De l'attention nait la motivation qui dépend des buts, des besoins, des valeurs et des plans du joueur. L'attention cognitive est définie par François Maquestiaux comme une ressource mentale mobilisée sur une ou plusieurs tâches (Maquestiaux, 2013, pp. 18-33). Certains arguments empiriques semblent démontrer des limitations structurales de l'attention sous la forme de goulet d'étranglement. Ce goulet d'étranglement fonctionnerait comme un barrage: la quantité d'eau écoulée, tout comme la quantité d'information, est gigantesque mais, à un moment donné, le débit d'eau, ou débit d'information, est réduit afin de pouvoir passer le barrage. Il s'agit d'une focalisation de l'attention sur un objet, une cible. Cette attention est stimulée soit par la cible soit par la décision de l'individu lui-même. L'objectif de l'artiste est de parvenir à ce que la cible stimule le spectateur afin de générer une conduite esthétique. Celle-ci est intentionnelle car dirigée vers un objet à propos duquel elle élabore du sens par le biais de représentations mentales. La conduite esthétique, explique Edmond Couchot, peut être dirigée vers un objet naturel ou un artefact et être source de plaisir ou de déplaisir (Couchot, 2012). Nous pensons que c'est en maîtrisant les processus d'attention cognitive que le chercheur en arts comprendra comment ménager des moments contemplatifs et éveiller l'attention d'un public non spécifiquement disposé, compte tenu des circonstances, à prêter attention à l'œuvre d'art. De ce fait, il ne suffit pas que l'attention du joueur soit disponible pour qu'il reçoive positivement l'œuvre qu'il a sous les yeux. Pour le dire autrement, il ne suffit pas de parachuter une vidéo expérimentale dans un jeu pour qu'elle soit contemplée, elle doit également être adaptée au contexte ludique dans lequel évolue le joueur. C'est ce qu'Hertzian Labyrinth, contrairement aux vidéos, est parvenu à faire.

Bernard Guelton avait déjà perçu que l'immersion dans une ARG pouvait être réelle, fictionnelle ou virtuelle (Guelton, 2012). Elle est réelle en termes sensorimoteurs, à condition que cessent les automatismes moteurs, elle est fictionnelle grâce à la Mimicry, enfin elle est virtuelle grâce à l'interactivité qui nous permet d'entrer et sortir du jeu à tout moment. Dans tous les cas, pour qu'il y ait immersion, une rupture est nécessaire. Si devant les vidéos expérimentales aucune rupture ne s'est opérée, les joueurs préférant s'en désintéresser, avec Hertzian Labyrinth, la rupture est présente dans tous les commentaires. Elle est sonore «C'est pour les épileptiques!» s'écrient les Tryharders dans la salle. Les joueurs de l'équipe des Soulards-Terriens renoncent à écouter le jeu avec le casque, «c'est une torture! ». Elle est esthétique pour les experts en art car les étudiants en Arts plastiques le rapprochent spontanément de l'«art psychédélique ». L'œuvre capte leur attention car elle est vitale pour le jeu, seule sa résolution permettra de connaître l'étape finale du parcours, en l'occurrence le Centre Pompidou Metz. L'immersion dans ce jeu interactif dure longtemps, entre dix et vingt 
minutes selon les équipes. Elle génère pour la première fois dans la salle des interactions entre joueurs voire une entraide. C'est, en effet, le seul moment où nous avons pu observer une coopération entre équipes adverses. Les Tryharders et les Moron Rabbits ayant compris le fonctionnement du jeu et étant sortis victorieux du labyrinthe, ils acceptent d'aider les M'Bonbons. Ils ne peuvent se retenir de déclarer : «Elles n'ont pas compris que ça doit faire un rond parfait ». Pour la première fois en quatre heures de jeu, la solidarité joue. Cette solidarité n'est pas uniquement due au jeu, elle tient aussi au moment du jeu, Hertzian Labyrinth intervenant à la toute fin d'Alter Ego. Les Tryharders et les Moron Rabbits collaborent avec les autres équipes parce qu'ils sont, à ce moment-là, hors compétition. On pouvait tout de même lire sur leur visage et dans leur comportement une certaine fierté d'être parvenu avant les autres équipes à maîtriser ce jeu d'où, certains, ne sortiront jamais.

\section{La transindividuation entre cohortes de joueurs}

18 Afin de mesurer au plus près le vécu en première personne des joueurs en ligne, j'ai proposé à l'ensemble des joueurs un questionnaire portant d'une part sur le moment fort du jeu, d'autre part sur les émotions ressenties. Ce questionnaire reprenait la question 33 du Questionnaire genevois d'appraisal ou QGA du psychologue Klaus Scherer. Les joueurs devaient décrire le moment du jeu qu'ils considéraient comme étant le plus fort et lui associer des émotions choisies parmi une liste de treize émotions, en les notant de 1 à 10 .

En 2013, les joueurs en ligne ayant répondu au questionnaire affirmaient que, pour eux, le moment fort du jeu n'était pas en lien avec leur performance sur Internet, mais correspondait à un événement survenu dans le monde tangible dont ils n'avaient pas été visuellement témoins. Ainsi, un internaute expliquait que le moment fort du jeu fut celui où il découvrit l'existence de l'auditorium au Centre Pompidou. Nous avions, en effet, caché l'un des indices dans un amphithéâtre à proximité de la salle informatique. Ce changement d'horizon, soit le passage du monde virtuel au monde réel, fut, comme nous l'espérions, générateur de surprise et d'émotions positives. Pour un autre internaute, le moment fort du jeu fut lié à un contretemps dans les rues de Metz, près de la galerie O'Cowbell, alors que ses partenaires en ligne le prévenaient de l'arrivée imminente de la milice. En définitive, pour les internautes, les moments forts du jeu n'étaient pas liés au monde numérique, c'est-à-dire à la résolution des énigmes ou à leur contact avec le maître du jeu, mais aux événements qui se passaient en-dehors de la salle. L'attention des internautes était donc bien tournée vers l'extérieur et vers leurs camarades.

En 2014, en revanche, les trois-quarts des joueurs en ligne déclarent majoritairement dans le questionnaire que le moment fort du jeu n'était pas en lien avec l'activité de leurs camarades dans la rue mais plutôt avec leurs propres performances sur le jeu en ligne. Cela peut s'expliquer par le fait que l'activité en ligne était beaucoup plus fouillée. Les joueurs devaient, sans l'aide de leurs camarades dans la rue, décrypter un texte grâce au code César, résoudre des mots croisés, retrouver des éléments du patrimoine historique de Metz sur le web, etc. Les entretiens, les observations de l'activité dans la salle et la lecture des textos échangés nuancent pourtant l'idée d'un manque d'interaction entre cohortes de joueurs. L'application installée sur le téléphone fourni par l'Université de Lorraine permettait aux joueurs sur le terrain d'adresser des 
textos à leurs camarades restés dans la salle informatique et le relevé des 407 textos envoyés par les Tryharders prouve qu'ils ont échangé constamment sur leur expérience du jeu. L'empathie est avérée pour cette équipe puisque ses internautes déclarent que pour eux le moment fort du jeu est en lien avec un événement survenu dans la rue dont ils n'ont pas été visuellement témoins, comme par exemple la chute de l'une de leurs camarades dans des cartons amoncelés dans la rue la plus étroite de Metz. Rappelons que l'empathie concerne notre capacité à nous mettre à la place d'autrui sans nécessairement ressentir ce qu'il éprouve. L'empathie, selon Jean Decety, a des éléments fondamentaux qui ne sont pas propres à l'être humain comme le mimétisme, la réciprocité et la communication des émotions (Decety, 2004, p. 60). L'empathie humaine contiendrait deux dimensions, la capacité de faire la différence entre soi et autrui et le partage des émotions avec des individus auxquels l'être humain s'identifie. La lecture des échanges de textes entre les deux cohortes de joueurs des Tryharders montre clairement que le contact entre eux a été permanent et qu'ils ont échangé des moments émotionnellement très forts. C'est grâce à la technologie que la transmission du vécu entre joueurs a été favorisée, donnant naissance à une esthésie collective du jeu où chaque participant assimile l'expérience d'autrui. L'exemple le plus marquant reste le moment où les joueurs sur le terrain écrivent à leurs partenaires en ligne qu'ils sont aux prises avec une bande d'adolescentes qui menacent une performeuse. Ils écrivent : " la ou on a fait la quete $2 \mathrm{y}$ a des meufs or compete qui veulent defoncer la cabane et des participants et on est les seules gars donc on reste avec mais tous les autres sont parti » (sic). Cette contagion du vécu entre joueurs nous autorise à analyser l'activité des joueurs à partir de leur expérience collective du jeu, la technologie ayant favorisé une transindividuation technique et psycho-sociale du jeu. Le terme «transindividuation », créé par Bernard Stiegler, est dérivé du terme «transindividuel " de Gilbert Simondon qui se distingue lui-même de l'interindividuel issu de la psychologie et de l'intrasocial en sociologie. Le psychologisme et le sociologisme, pour reprendre les termes de Simondon, sont insuffisants pour traduire, selon lui, ce qu'il nomme la réalité psycho-sociale car dans l'inter-individuel «ce sont les individus qui font le groupe » et dans l'intra-social « c'est le groupe qui fait les individus » ${ }^{7}$.

Pour Simondon, seul le transindividuel est susceptible de traduire l'individuation psychique et collective qui « rompt avec l'individuation vitale, et où l'individu vivant se prolonge et se dépasse : dans cette nouvelle forme d'individuation indissociablement psychique et sociale, le "collectif réel" n'est ni la simple réunion de psychismes individuels déjà donnés, ni le "social pur" des insectes: c'est un devenir social qui s'individue en "unité collective" parallèlement à la "personnalisation" singulière de chaque sujet psychique. $»^{8}$ Bernard Stiegler est l'un des principaux héritiers de la pensée simondienne. Il a repris ces préoccupations qu'il a prolongées à l'ère d'Internet. Selon lui, il y a transindividuation car les êtres humains sont des êtres techniques et symboliques qui ne s'individuent qu'avec l'autre (Stiegler, 2005, p. 63). Nous ne sommes qui nous sommes, explique-t-il, qu'en nouant avec autrui une relation dans laquelle nous le transformons en même temps que nous nous transformons. Pour contredire autrui, poursuit-il, nous fabriquons des arguments qui transforment sa pensée ainsi que le milieu dans lequel nous évoluons avec cet autre. 


\section{Les joueurs, milieu associé du jeu} au lieu de le subir. Plusieurs modalités d'action témoignent de cette transformation du milieu technique. La première consiste à acculer l'organisateur du jeu à modifier le jeu pendant son activation, la deuxième consiste à enrichir la technologie du jeu en devenant contributeur du jeu. Ainsi, l'un des moments les plus intéressants du jeu est celui où les joueurs déstabilisent les organisateurs, les amenant à improviser et imaginer des intrigues de dernière minute. En 2013, pour réduire les écarts de temps trop importants, le maître du jeu avait décidé d'une part d'occuper l'équipe d'informaticiens en les mettant sur de fausses pistes, et d'autre part de résoudre certaines énigmes à la place de l'équipe de plasticiens. En d'autres termes, nous avions parfois perdu le contrôle de la situation. En 2014, une équipe de joueurs composée d'étudiants en informatique a testé les limites du jeu en injectant des requêtes SQL $^{9}$ dans les différents champs de texte du site. Ces débordements ne furent pas propres à Alter Ego. Par exemple, l'un des joueurs de The Beast, le jeu créé par Microsoft en 2001, raconte la chose suivante: "À mesure que nous réussissions de mieux en mieux à résoudre les énigmes, il leur fallait en inventer des plus difficiles [...] À un moment, nous avons même trouvé dans leur code source des choses dont ils ignoraient la présence. Il a fallu qu'ils inventent une nouvelle histoire pour les couvrir » (cité par Jenkins, 2013, p. 161).

En 2014, nous avons tenté d'augmenter le niveau de difficulté des énigmes et fourni des indices en ligne qui consommaient, à chaque utilisation, cinq diamants. Les équipes constituées par des étudiants en informatique, familiarisés avec les raisonnements logiques, prennent chaque année plus d'avance sur les équipes d'étudiants en sciences humaines. Ils développent avant le début du jeu une stratégie particulière : les Moron Rabbit n'ont laissé qu'une personne devant le poste informatique, alors que les Tryharders sont venus avec leur propre ordinateur potable, en plus du poste mis à leur disposition. C'est là que s'est manifestée la seconde modalité d'action sur le milieu technique. J'ai en effet pu apprendre, à la lecture des échanges de textos, que les Tryharders avaient créé pour le jeu ce qu'ils dénomment entre eux le «pad $»^{10}$. Il s'agit d'un logiciel en ligne permettant d'effectuer des copier-coller sur le web et de télécharger le tout sur les smartphones. Ce n'est plus alors Internet qui est le « milieu associé » des joueurs mais les joueurs qui en sont le milieu associé. Le concept de milieu associé a été inventé par Simondon pour désigner un milieu technique particulier, tel l'objet technique et le milieu naturel auquel il est lié de façon indissociable (Simondon, 1958). C'est par exemple le cas, explique Simondon, des usines marémotrices où l'eau de mer fournit l'énergie nécessaire, rend étanches les paliers par la pression de l'eau et refroidit les turbines. Cette notion peut s'étendre à l'homme. Pour Simondon, l'apprentissage au moyen duquel un homme forme des habitudes, des gestes qui lui permettent de se servir d'outils, pousse cet homme à s'individualiser techniquement. C'est lui, dès lors, qui devient milieu associé de ces divers outils. Pour Stiegler, le réseau Internet associe de même l'élément humain à un milieu technique. Le site de l'association Ars industrialis propose la définition suivante du milieu associé : «Un milieu techno-symbolique vous est associé s'il est le medium et le vecteur de votre individuation, celle-ci n'étant possible que parce que ce milieu associe des individus ${ }^{11}$. " L'article précise plus loin: «La nouveauté du réseau Internet en tant que milieu 
technique, par contraste avec la télévision par exemple, est qu'il ne constitue pas un milieu structurellement dissocié. Telle est la raison pour laquelle Internet rend possible l'économie contributive, typique du logiciel libre. Il n'y a plus dissociation des producteurs et des consommateurs, mais association des destinataires et des destinateurs produisant une nouvelle forme de socialité et un nouvel esprit du capitalisme. " Ainsi, par le retournement qu'elle opère entre objets et individus, la pensée de Simondon permet de penser la réception de l'œuvre interactive. Les individus constituant le milieu associé dans lequel évoluent les objets techniques, il devient alors pertinent d'établir l'expérience esthétique comme trace de ce milieu.

\section{Conclusion}

Alter Ego est un exemple de jeu artistique à réalité alternée tourné vers la médiation, prise dans le double sens de relation singulière au monde et d'intermédiaire. La gamification tenterait alors de fonder une expérience du vivre ensemble en s'appuyant sur le web et sur les processus de transindividuation. Pour Bernard Stiegler, le public est devenu grand public, celui du désert qui croît et où se répand la misère symbolique. La solution consisterait alors à réinventer la figure de l'amateur (Stiegler, 2005, p. 33) où l'écoute, la contemplation et la création de l'œuvre interagiraient étroitement. Les jeux artistiques à réalité alternée pourraient participer à la réinvention de cette figure de l'amateur en sensibilisant ses participants à l'art contemporain. Alter Ego, en particulier, transforme les spectateurs en contributeurs en leur proposant de manipuler les œuvres et en leur laissant un espace de liberté, espace dans lequel ils peuvent améliorer le milieu technique auquel ils sont associés.

Toutefois, les résultats de la recherche montrent que si l'ARG artistique souhaite transformer la figure du joueur en figure de l'amateur, le joueur, en retour, contraint l'objet artistique à s'hybrider avec le milieu technique dans lequel il veut s'intégrer. Des vidéos expérimentales parachutées telles quelles dans l'univers ludique ne fonctionnent pas dans la mesure où elles ne rencontrent pas leur public. Il se pourrait qu'en ménageant davantage de temps de pause aux joueurs en ligne, ceux-ci passent davantage de temps à les visionner. Mais dès lors, il s'agirait de combler un temps vide et, par dépit, d'inciter les joueurs à contempler des vidéos, pour passer le temps. Cette modalité contemplative ne semble pas satisfaisante. Les vidéos expérimentales doivent plutôt devenir les éléments vitaux de l'ensemble auquel elles veulent s'intégrer. L'objet artistique ayant le mieux fonctionné dans cette étude est un objet hybride entre l'objet artistique, l'objet ludique et l'objet technique. Hertzian Labyrinth est objet artistique, il s'agit d'une œuvre créée par un artiste, exposée dans un contexte artistique durant la résidence de l'artiste à Valenciennes. La visée esthétique du jeu, selon l'artiste, est d'agir sur les compétences d'écoute des joueurs. Hertzian Labyrinth trouve de plus un certain succès auprès des joueurs experts en art qui reconnaissent en lui un style psychédélique. Le procédé de l'œuvre est par ailleurs ludique. Il comprend des règles ouvrir des portes en cliquant sur la souris -, un but clair - sortir du labyrinthe - mais le feedback est totalement absent du jeu si bien qu'un joueur finira par demander à la salle si quelqu'un était parvenu à sortir du jeu. Hertzian Labyrinth est enfin une démonstration des possibilités techniques de Processing, un logiciel développé par deux chercheurs du MIT, Ben Fry et Casey Reas, à destination des artistes. Derrière l'outil se cache des savoir-faire humains et une histoire du codage qui permet d'inscrire 
l'objet technique dans le domaine culturel. Selon moi, c'est vers ce type d'objet hybride qu'Alter Ego devrait désormais se diriger. Cela n'exclut pas d'agir par ailleurs sur le contexte du jeu afin de favoriser l'éveil de moments contemplatifs, en abandonnant notamment l'idée d'une course-poursuite au profit d'un temps maximal imparti aux joueurs.

Des questions de recherche restent toutefois en suspens. Les joueurs non experts en art auront-ils été sensibilisés à l'art numérique ? Cette expérience aura-t-elle une incidence sur leur sensibilité aux œuvres interactives? Pour le mesurer, nous devrions suivre un groupe témoin de joueurs non experts en art sur plusieurs années. Or, à ce jour, l'anonymat des questionnaires ne nous a pas permis d'effectuer un tel suivi. Ce suivi pourrait être facilement envisagé grâce à la mise en place d'une collaboration de recherche avec un enseignant en IUT qui aurait plus facilement la possibilité de suivre les joueurs sur au moins deux années d'études.

\section{BIBLIOGRAPHIE}

BAILLY R. (2001), « Le jeu dans l'œuvre de D.W. Winnicott », Enfances \& Psy, n 15, pp. 41-45.

CAILLOIS R. ([1958]1992), Les jeux et les hommes, Paris, Gallimard.

COUCHOT E. (2012), La Nature de l'art: Ce que les sciences cognitives nous révèlent sur le plaisir esthétique, Paris, Hermann.

DECETY J. (2004), « L'empathie est-elle une simulation mentale de la subjectivité d'autrui », in A. Berthoz (dir.), L'empathie, Paris, Odile Jacob, pp. 53-88.

GROUPIERRE K. et LELIEVRE E. (2013), « "Les Mystères de la Basilique” - Plaisir fictionnel, ludique et esthétique au service du patrimoine», Culture numérique, en ligne : http://

culture.numerique.free.fr/publications/ludo12/lelievre_ludovia_2012.pdf

GUELTON B. (2012), « Représentations de soi et immersions dans les réalités alternées Dispositifs ludiques et fictionnels ", Interfaces numériques, Volume 1 - n² 2, pp. 55-71.

JENKINS H. (2013), La culture de la convergence : Des médias au transmédia, Paris, Armand Colin.

LELIEVRE E. (2012), Des jeux de rôle en ligne tridimensionnels aux jeux à réalité alternée : expérience esthétique, création et expérimentation, thèse de doctorat, Université Paris 8 Saint-Denis.

MAQUESTIAUX F. (2013), Psychologie de l'attention, Bruxelles, De Boeck.

McGONIGAL J. (2011), Reality is Broken: Why Games Make Us Better and How They Can Change the World, London, Ed. Vintage digital.

SIMONDON G. ([1958]2012), Du mode d'existence des objets techniques, Paris, Aubier.

SIMONDON G. (2013), Cours sur la perception [1964-1965], Paris, PUF.

STIEGLER B. (2005), De la misère symbolique, Paris, Flammarion.

STIEGLER B. (2008), Prendre soin. De la jeunesse et des générations Tome 1, Paris, Flammarion. 
TISSERON S. (2013), Du livre et des écrans : Plaidoyer pour une indispensable complémentarité, Paris,

Manucius.

\section{NOTES}

1. «Naches, mot yiddish qui décrit la fierté éclatante ressentie lorsque quelqu'un à qui l'on a enseigné ou duquel on est le mentor connaît une réussite, classé juste derrière la surprise et le fiero.» Traduit par nos soins: «Naches, a Yiddish word for the bursting pride we feel when someone we've taught or mentored succeeds, ranked just below surprise and fiero. "

2. " "Games are an obvious source of flow," he wrote, "and play is the flow experience par excellence." " D'après M. Csíkszentmihályi (1975), Beyond Boredom and Anxiety: The Experience of Play in Work and Games, San Francisco, Jossey-Bass, 1975, p. 37.

3. Cet enregistrement a fait l'objet d'une déclaration à la Commission nationale de l'informatique et des libertés (CNIL) en France.

4. http://www.affective-sciences.org/system/files/webpage/GAQ_Fran\%C3\%A7ais_0.pdf

5. Traduction libre : « My view of the performance was clouded by the terror, frustration, boredom and fury that dominated my 24 hours in captivity. [..] Certainly, no other performance I have ever seen has brought about such intense extremes of emotion. ", en ligne: http://blasttheory.co.uk/bt/ work_kidnap.html

6. https://vimeo.com/62497784

7. http://arsindustrialis.org/vocabulaire-ars-industrialis/transindividuation

8. Idem.

9. Structured Query Language, c'est-à-dire un langage informatique de requête structurée.

10. http://titanpad.com/alterego

11. http://arsindustrialis.org/milieu

\section{RÉSUMÉS}

Je propose, à travers une étude de cas, celle du jeu artistique à réalité alternée Alter Ego, d'analyser la réception de ce type de jeu par des joueurs en ligne. Après avoir rappelé l'apport du collectif britannique Blast Theory dont Alter Ego s'inspire, j'aborderai les ressorts de l'immersion fictionnelle et virtuelle du jeu. En m'appuyant sur une enquête sur les émotions, des entretiens, des observations en présentiel et filmées, une analyse des textos échangés, je tenterai de confirmer la dimension artistique de ce jeu qui consiste d'une part à éveiller des moments contemplatifs, d'autre part à partager, quatre heures durant, une aventure relationnelle. En outre, en m'appuyant sur les concepts simondiens de transindividuel et de milieu associé, je tenterai d'expliquer la façon dont se coconstituent l'objet technique et le joueur.

I propose, here in, to analyze Alter Ego, an artistic alternated reality game, from the online gamers'point of view. I'll first present Blast Theory's work which inspired Alter Ego, and then present the keys of virtual and fictional immersion. Based on an emotional inquiry, interviews, observations, analysis of short messages, I will try to confirm that the artistic dimension of this game consists first on the arousal of contemplation, secondly in a relational adventure. In 
addition, relying on Simondon's concepts, I'll try to explain how the technical object and the player are linked together as a whole.

INDEX

Mots-clés : jeu, réalité alternée, transindividuation, réception, technologie

Keywords : game, alternated reality, transindividuation, artistic reception, technology

\section{AUTEUR}

FRANÇOISE LEJEUNE

Université de Lorraine 